\title{
Effects of screening and brief intervention on alcohol consumption in an emergency department
}

\author{
Soo Chul Im, Duk Hee Lee \\ Department of Emergency Medicine, Ewha Womans University College of Medicine, Seoul, Korea
}

Objective Alcohol use is associated with high levels of morbidity and mortality. Alcohol problems are common in emergency departments (EDs). This study investigated the effect of screening and a new brief intervention (BI) protocol on alcohol consumption of ED patients.

Methods The participants of this study were those aged 18 years or older who visited the ED due to injury over 12 weeks. BI was offered to patients with a score of 8 or higher on alcohol use disorders identification test (AUDIT) screening. Follow-up telephone assessments were conducted at one week, one month, and three months.

Results The risk drinker (RD) group (AUDIT 8-15) comprised 101 patients, and the alcohol use disorder (AUD) group (AUDIT > 16) comprised 41 patients. Before the BI, the weekly mean alcohol intake amount for the RD group was $180.90 \pm 98.34 \mathrm{~g}$ and for the AUD group was $358.00 \pm$ $110.62 \mathrm{~g}$. Alcohol consumption was reduced to $132.39 \pm 75.87 \mathrm{~g}$ in the RD group and $181.86 \pm$ $78.11 \mathrm{~g}$ in the AUD group in the 3-month follow-up assessment. Alcohol consumption in the AUD group reduced significantly compared to the $\mathrm{RD}$ group $(\mathrm{P}<0.001)$.

Conclusion Alcohol screening and $\mathrm{BI}$ contributed to alcohol intake reduction in ED patients. Specifically, the BI effect was greater in the AUD group than the RD group. The ED can be an effective place to begin implementing screening and intervention for alcohol use patients at risk.

Keywords Alcoholism; Brief intervention; Emergency service, hospital

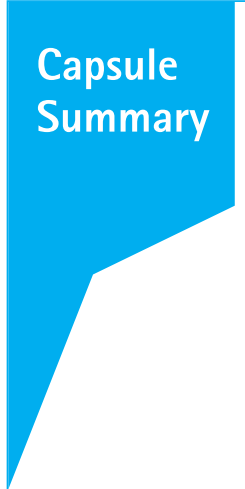

What is already known

In developed countries, the alcohol screening and appropriate intervention are being performing. A brief intervention has been shown to be cost-effective in reducing alcohol consumption and alcohol-related harm in ED patients.

What is new in the current study

A brief intervention reduced alcohol consumption in ED patients with alcohol use disorders and risky drinking. The ED is an ideal site for brief intervention in these patients.
elSSN: 2383-4625

Received: 18 September 2019 Revised: 20 December 2019

Accepted: 2 March 2020

Correspondence to: Duk Hee Lee Department of Emergency Medicine, Ewha Womans University College of Medicine, 1071 Anyangcheon-ro, Yangcheon-gu, Seoul 07985, Korea E-mail: calla@ewha.ac.kr ORCID

https://orcid.org/0000-0001-5992-5284

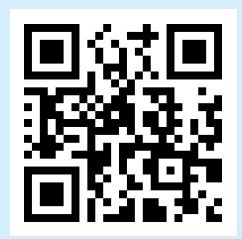

How to cite this article:

Im SC, Lee DH. Effects of screening and brief intervention on alcohol consumption in an emergency department. Clin Exp Emerg Med 2020;7(4):310-318. https://doi.

org/10.15441/ceem.19.080

This is an Open Access article distributed under the terms of the Creative Commons Attribution Non-Commercial License (https:// creativecommons.org/licenses/by-nc/4.0/). 


\section{INTRODUCTION}

Alcohol consumption continues to be a significant contributing factor to the hospital admission and death. ${ }^{1}$ Alcohol is the most commonly used and abused substance in the United States, accounting for one in 10 deaths among adults between ages 20 and $64 .{ }^{2}$ The number of emergency department (ED) visits related to alcohol use has increased in the United States (approximately five million alcohol-related visits by 2014) costing 15.3 billion dollars. $^{3}$

South Korea has a liberal drinking culture and people have limited awareness of alcohol disorders; this is a major barrier to improving drinking habits. The socioeconomic cost of drinking has been increasing in South Korea. Compared to 2005, cost increased by 2.3 times in 2013. ${ }^{4}$ By 2018, the South Korean drinking population comprised $65.2 \%$ of the entire population. The proportion of high-risk drinkers is approximately $18.5 \% .{ }^{5}$ The lifelong prevalence of alcohol use disorder (AUD) in South Korea is approximately $13.9 \% \%^{6}$; the estimated number of patients with AUD is 1.39 million people. ${ }^{7}$

Alcohol misuse is a major problem confronting all EDs. ${ }^{8}$ The National Institute on Alcohol Abuse and Alcoholism suggests that physical examination, administration of drugs that interact with alcohol, ED visits, and outpatient visits for alcohol-related disease are promising approaches to screen patients with AUD. ${ }^{9}$ Some studies have reported that alcohol intake behavior could be changed by screening patients and conducting a brief motivational intervention in unique situations such as when injuries occurred recently. ${ }^{10,11}$ In a comparative analysis involving 6,000 patients from 32 control studies, Bien et al. ${ }^{12}$ found that the treatment effect of brief motivational interventions was greater than that of extensive treatment. Alcohol screening and brief intervention is an effective and cost-effective method to lower consumption levels and reduce alcohol-related harm among ED patients. ${ }^{13}$

South Korean patients self-reporting or suspected of alcohol use accounted for $19.8 \%$ of total ED visits in one study. ${ }^{14}$ However, screening and intervention for such patients who visit EDs and other hospital departments are not routinely implemented in South Korea. Therefore, this study investigated the effect of screening and brief intervention (BI) on ED patients.

\section{METHODS}

\section{Design}

Physicians performed the alcohol screening with alcohol use disorders identification test (AUDIT) (Appendix 1) for 12 weeks to ED patients who visited for injuries. The study participants were aged
18 years or older. If the patients had an AUDIT screening score of 8 or more, patients were provided Bl. We then conducted telephone follow-up assessments at one week, one month, and three months. During the 1-week follow-up assessment, the patients were asked their mean weekly frequency of alcohol consumption, intake amount, and will to change their drinking habit. During the 1- and 3-months follow-up assessments, they were asked about their mean weekly frequency of alcohol consumption and intake amount; they were then asked to self-assess their success level in achieving their alcohol abstinence goal and their AUDIT-C scores. The AUDIT-C is the screening tool measuring recent intention of drinking, including questions 1 to 3 of AUDIT.

We excluded those who were hospitalized, transferred to other hospitals, dead, fully drunk, or medically unstable at the time of the visits.

\section{AUDIT}

The Korean version of AUDIT is now widely used for alcohol counseling in the second checkup of the Life Transition Period Health Examination, a national health management project by the Ministry of Health and Welfare of South Korea. Four ED interns during this research period conducted the AUDIT for injured patients. Two groups of two interns took turns working 24-hour shifts; they performed their daily tasks in the ED concurrently.

\section{Classification of drinker groups}

Those with an AUDIT score of 0 to 7 were classified as the normal drinker group. Those with an AUDIT score of 8 to 15 were classified as the risk drinker (RD) group. ${ }^{15}$ Risk drinking is drinking behavior that causes mental, social, and legal problems; it increases the risks of various diseases including hypertension, gastrointestinal bleeding, sleep disorders, major depression, strokes, cirrhosis, and malignant tumors. ${ }^{16}$ AUD was defined as scoring 16 points or higher.

\section{Development and implementation of the BI protocol}

We developed a BI protocol based on National Institute on Alcohol Abuse and Alcoholism guidelines, literature review, and an algorithm of the Academic Emergency Department Screening, Brief Intervention, and Referral to Treatment Collaborative. ${ }^{17}$ In the first step, patients were informed of screening results. They were told that the RD group is subject to various health problems and informed of recommended alcohol intake amounts. They were then asked about the relationship between the cause of admission and drinking. A patient-centered counseling technique encouraged them to express their opinions of the relationship and summarized the patients' remarks empathically. To promote self- 
motivation in the second step after listening to their opinions about the pros and cons of alcohol consumption, they were asked to measure their determination and level of willingness in abstaining from alcohol. Finally, in the third step of the protocol, the patients were encouraged to identify their specific goals for abstinence from alcohol. In addition, if the patients agreed, they signed a letter of agreement on abstinence (Appendix 2). The developed protocol was reviewed by an expert on counseling and intervention.

This study provided education and training on interviewing and intervention to the ED medical staff in charge of conducting the $\mathrm{BI}$. Four doctors and 12 nurses volunteered to participate in the study with three researchers. Five 3-hour programs were attended by the medical staff members. Those who completed the BI education conducted the BI for patients who had AUDIT scores of 8 points or higher.

\section{Ethics statement}

The study procedure was approved by the institutional review board of Ewha Womans University Mokdong Hospital (17-09059). The AUDIT and the BI were conducted after obtaining patient consent. If the patients refused our first request for a telephone follow-up assessment, no further telephone follow-up assessment was conducted. There were no financial incentives for the participants.

\section{Statistical analysis}

Non-continuous variables were cross-tabulated using PASW Statistics ver. 18 (SPSS Inc., Chicago, IL, USA). Student's t-test com-

Table 1. Patients who underwent the AUDIT screening test

\begin{tabular}{cccccc}
\hline & & \multicolumn{4}{c}{ AUDIT (score) } \\
\cline { 3 - 6 } & & $\begin{array}{c}\text { Normal } \\
(0-7)\end{array}$ & $\begin{array}{c}\text { Risk drinker } \\
(8-15)\end{array}$ & $\begin{array}{c}\text { AUD } \\
(\geq 16)\end{array}$ & Total \\
\hline Drinking & Yes & 58 & 54 & 25 & 137 \\
alcohol & No & 432 & 98 & 34 & 564 \\
& Total & 490 & 152 & 59 & 701 \\
\hline
\end{tabular}

AUDIT, alcohol use disorders identification test; AUD, alcohol use disorder.

Table 2. Brief intervention non-participation reasons

\begin{tabular}{llc}
\hline & \multicolumn{1}{c}{ Reason } & Value \\
\hline Patient & Refusal & $37(53.3)$ \\
& Admission and transfer & $2(2.9)$ \\
Medical staff & Confusion of the target group & $4(5.8)$ \\
& Busy or absence of staff & $25(35.9)$ \\
& Others & $1(1.9)$ \\
Total & & $69(100)$ \\
\hline
\end{tabular}

Values are presented as number (\%). pared continuous variables. Repeated measure analysis of variance was used for alcohol consumption change difference in the $\mathrm{RD}$ and AUD groups. P-value less than 0.05 indicated statistical significance.

\section{RESULTS}

The number of patients screened was 701. The mean age of the AUDIT participants was $35.70 \pm 11.05$ years old; 411 male participants' (58.6\%) mean age was $35.32 \pm 11.32$ and 290 female participants' (41.4\%) mean age was $38.22 \pm 11.98$. Of those, 137 patients (17.7\%) self-reported or were suspected of alcohol use within 6 hours and 564 patients (82.3\%) were not. The RD group comprised 152 patients and the AUD group comprised 59 (Table 1).

BI was provided to 142 patients. Of those, 69 patients did not participate in the intervention. Specifically, 39 patients could not receive intervention counselings because of patient-associated reasons such as the refusal, hospitalization, or transfer; the remaining 30 patients could not participate because of medical staff-associated reasons such as the lack of study-trained medi-

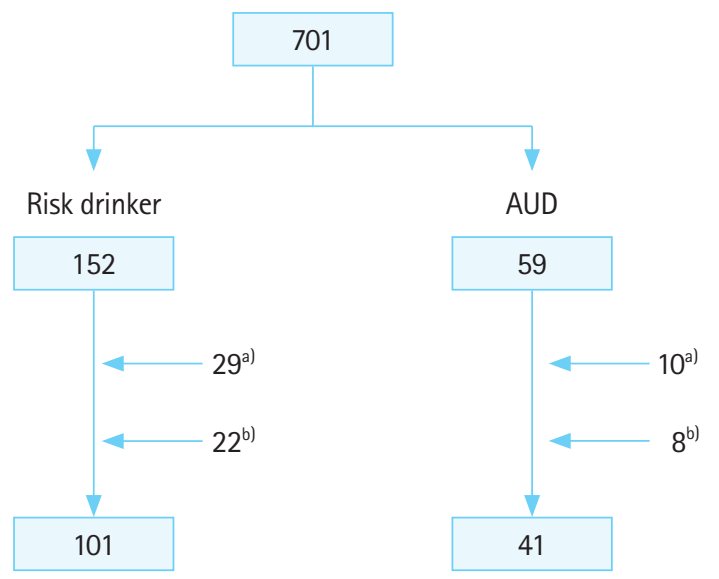

Fig. 1. Study population flowchart. AUD, alcohol use disorder. ${ }^{\text {a) }}$ Patientassociated reasons (refusal of intervention, hospitalization, or transfer). ${ }^{b}$ Medical staff-associated reasons (the lack or absence of medical staff who completed the education program at the time of the patients' visit to the emergency department).

Table 3. Comparison of the $\mathrm{BI}$ and non-Bl groups

\begin{tabular}{|c|c|c|c|c|}
\hline & $\begin{array}{l}\text { BI group } \\
(n=142)\end{array}$ & $\begin{array}{l}\text { Non-Bl group; } \\
\text { patient-associat- } \\
\text { ed reasons } \\
(n=39)\end{array}$ & $\begin{array}{l}\text { Non-Bl group; } \\
\text { medical staff-as- } \\
\text { sociated reasons } \\
\quad(n=30)\end{array}$ & P-value \\
\hline Age (yr) & $34.97 \pm 11.06$ & $35.60 \pm 10.21$ & $36.10 \pm 11.30$ & 0.331 \\
\hline Male & 92 (64.8) & 24 (61.5) & 19 (63.3) & 0.753 \\
\hline Drinking rate & $62(43.7)$ & $10(26.3)$ & 7 (22.6) & 0.074 \\
\hline
\end{tabular}

Value are presenged as number (\%) or mean \pm standard deviation. $\mathrm{BI}$, brief intervention. 
Table 4. BI group analysis

\begin{tabular}{|c|c|c|c|c|}
\hline & Risk drinker $(n=101)$ & AUD $(n=41)$ & Total $(n=142)$ & P-value \\
\hline Age (yr) & $35.16 \pm 12.12$ & $29.81 \pm 11.23$ & $33.97 \pm 12.06$ & 0.118 \\
\hline Male & $62(61.4)$ & $30(73.2)$ & $92(64.8)$ & 0.775 \\
\hline Mean BI time (min) & $8.96 \pm 3.02$ & $8.81 \pm 2.70$ & $8.93 \pm 2.93$ & 0.857 \\
\hline Current weekly alcohol intake $(\mathrm{g})$ & $180.90 \pm 98.34$ & $358.00 \pm 110.62$ & $195.83 \pm 97.36$ & 0.007 \\
\hline Drinking abstinence goal (g) & $56.30 \pm 61.50$ & $108.75 \pm 95.84$ & $67.96 \pm 73.16$ & 0.053 \\
\hline Will to reduce drinking (0-10 points) & $6.05 \pm 2.31$ & $6.06 \pm 2.32$ & $6.06 \pm 2.30$ & 0.989 \\
\hline
\end{tabular}

Values are presented as mean \pm standard deviation or number (\%).

$\mathrm{BI}$, brief intervention; AUD, alcohol use disorder.

Table 5. BI results according to the drinking group

\begin{tabular}{lccc}
\hline & $\begin{array}{c}\text { Risk drinker } \\
(\mathrm{n}=101)\end{array}$ & $\begin{array}{c}\text { AUD } \\
(\mathrm{n}=41)\end{array}$ & P-value \\
\hline Mean BI time (min) & $8.96 \pm 3.02$ & $8.81 \pm 2.69$ & 0.857 \\
Average alcohol intake/wk (g) & & & \\
1 wk before BI & $180.90 \pm 98.34$ & $358.00 \pm 110.62$ & 0.007 \\
Drinking abstinence goal & $56.30 \pm 61.50$ & $108.75 \pm 95.85$ & 0.053 \\
1 wk & $7.89 \pm 10.25$ & $50.77 \pm 34.59$ & 0.110 \\
1 mo & $76.06 \pm 49.23$ & $156.67 \pm 98.70$ & 0.081 \\
3 mo & $132.39 \pm 75.87$ & $181.86 \pm 78.11$ & 0.864 \\
AUDIT score & & & \\
At the time of BI & $9.80 \pm 2.70$ & $20.63 \pm 3.32$ & $<0.001$ \\
AUDIT-C score & & & \\
At the time of BI & $7.59 \pm 1.53$ & $9.31 \pm 1.58$ & 0.001 \\
1 mo & $3.02 \pm 2.76$ & $5.00 \pm 3.89$ & 0.034 \\
3 mo & $4.95 \pm 3.80$ & $4.45 \pm 3.20$ & 0.697 \\
\hline
\end{tabular}

Values are presented as mean \pm standard deviation.

$\mathrm{BI}$, brief intervention; AUD, alcohol use disorder.

cal staff at the time of the patients' ED visit (Table 2). There were no significant differences in the age, sex ratio, or alcohol intake percentage between the non- $\mathrm{Bl}$ and $\mathrm{BI}$ groups (Table 3).

There were 142 patients analyzed (Fig. 1). There was no significant difference in intervention time between the RD and AUD groups. The mean intervention time per patient was approximately $8.93 \pm 2.93$ minutes (RD group $8.96 \pm 3.02$ minutes, AUD $8.81 \pm 2.70$ minutes). The total weekly mean alcohol intake amount was around $195.83 \pm 97.36 \mathrm{~g}$ at one week before the BI (Table 4).

The weekly mean alcohol intake of the RD group was $180.90 \pm$ $98.34 \mathrm{~g}$ before the $\mathrm{Bl}$, which was reduced to $132.39 \pm 75.87 \mathrm{~g}$ by the 3-month follow-up assessment. The weekly mean alcohol intake of the AUD group was $358.00 \pm 110.62 \mathrm{~g}$ before the $\mathrm{Bl}$, which was reduced to $181.86 \pm 78.11 \mathrm{~g}$ by the 3-month follow-up assessment (Table 5).

The mean AUDIT-C score of the RD group was $7.59 \pm 1.53$ at the time of the $\mathrm{Bl}, 3.02 \pm 2.76$ in the 1-month follow-up assessment, and $4.95 \pm 3.80$ in the 3-month follow-up assessment. The mean AUDIT-C score of the AUD group was $9.31 \pm 1.58$ at the time of the $\mathrm{BI}, 5.00 \pm 3.89$ in the 1-month follow-up assessment, and
$4.45 \pm 3.20$ in the 3-month follow-up assessment (Table 5). Both groups demonstrated significant reductions of consumption alcohol. The reduction in alcohol consumption was significantly greater $(\mathrm{P}<0.01)$ in the AUD group compared to the RD group.

\section{DISCUSSION}

The ED can potentially provide early intervention for RD and AUD patients with simple examination tools. ${ }^{18}$ In South Korea, there are no alcohol reduction systems or programs in general hospitals. This study was the first attempt to screen and intervene with alcohol-related patients in South Korean ED.

A study by van Loon et al. ${ }^{19}$ reported that $9.8 \%$ of ED patients had an AUDIT score of 8 or higher. In this study, 30.1\% had an AUDIT score of 8 or higher. The mean AUDIT score at the time of the $\mathrm{BI}$ was $7.97 \pm 1.695$, reduced to $3.51 \pm 3.16$ (normal drinker level) at the 1-month follow-up assessment. The AUDIT score was increased to $4.81 \pm 3.51$ at the 3-month follow-up assessment but remained within the normal drinker level. Therefore, the $\mathrm{BI}$ effect may last for more than three months. However, this study evaluated the intervention effect by monitoring the abstinence success rate through follow-up observations. The results showed that the abstinence success rate was reduced from 58 patients $(90.8 \%)$ at 1 week to 48 patients $(82.8 \%)$ at 1 month and then to 18 patients (37.5\%) at 3 months; thus, an increasing number of patients failed to maintain abstinence. This finding suggested that the $\mathrm{Bl}$ effect weakens over time and additional interventions should be considered to maintain the abstinence effect. In addition, it may be necessary to develop methods to link the BI with a community-run abstinence program for AUD patients. Of 16 patients in the study considered to be in need of community-run abstinence programs, 15 patients refused our request to sign the letter of consent, resulting in a high refusal rate. To address this issue, education to increase patient awareness about the importance of continuous management and an offer a communitylinked intervention program to reduce patient burden and refusal is necessary. 
The main purpose of the $\mathrm{BI}$ is to reduce overall consumption and harmful activities such as binge drinking. Authoritative resources on this issue have already been established and can be used for people with risky or harmful drinking behavior, which are currently available to non-specialists. ${ }^{20,21}$ This study confirmed the necessity and effectiveness of alcohol intervention in South Korea. Furthermore, this study could change the perception of medical staff toward intervention by providing education on BI.

Several studies have reported different results on varied brief intervention effects between the two groups. A study by Barata et al. ${ }^{22}$ analyzed 37 studies involving the SBIRT (Screening, Brief Intervention, and Referral to Treatment) method with ED patients and found a small reduction in alcohol intake in low or moderate drinker groups. Among these studies, 35 studies were conducted in the United States, one was conducted in the UK, and one was conducted in Australia. The studies reported that emergency practitioners failed to achieve statistically significant differences in terms of brief intervention effects. ${ }^{18,23}$ In contrast, this study found that the RD group showed decreased alcohol intake and three month follow-up observation demonstrated that the reduction of alcohol intake was greater in the AUD group than in the RD group.

This study developed a brief alcohol intervention program for injured ED patients; the protocol was reviewed by a counseling and intervention professional. In addition, the intervention program was implemented in a medical setting by educating medical staff. To ensure that this intervention is used in EDs, ED medical staff awareness needs to be addressed; some problems were also found in the process of this study.

One limitation of this study is the limited number of patients who were offered Bl. A large number of patients were lost in the screening process because there were no additional staff members to administer the screening test and intervention program. The AUDIT implementation rate was relatively low overnight and on weekends when the ED was overcrowded. This may be a limitation because patients with alcohol tend to visit the ED overnight and on weekends. One report suggested one in three adult ED patients consume alcohol prior to presentation and the number rises to two in three after midnight. ${ }^{8}$ Another selection limitation was the participation of ED interns to screen potential patients. To improve AUDIT use, additional staff members need to be recruited or more consistent self-report screening may be implemented. In addition, this study was conducted at a single institution; a multicenter study needs to be conducted in the future. Patient socioeconomic status should also be studied because brief intervention effects can differ.

As reported by other studies, high costs were required for the
SBIRT approach. However, some studies reported that the ED intervention was far more cost-effective than the standard or outpatient care. ${ }^{24,25}$ As a result, brief intervention programs for RD patients should be implemented in South Korean EDs. Other study limitations are the lack of resources and unwillingness to implement or conduct total or partial abstinence programs. Additional systemic and long-term policies need to be established to address these limitations.

In summary, the ED can be an effective place to begin implementing screening and intervention programs for potential RD patients. AUDIT use and the BI contributed to reduction of alcohol intake of ED patients. In particular, the BI effect was greater on the AUD group than the RD group.

\section{CONFLICT OF INTEREST}

No potential conflict of interest relevant to this article was reported.

\section{ACKNOWLEDGMENTS}

This work was supported by the National Research Foundation of Korea (NRF) grant funded by the Korea government (MSIT) (No. 2018R1C1B5046096).

We thank Hey Ah Lee, PhD for support with the statistical analysis and Koo Young Jung, MD, PhD for conceptualization.

\section{REFERENCES}

1. Wisniacki F. Taking control of alcohol-related emergency department visits. Emerg Med J 2018;35:74.

2. Fernandez $A C$, Waller $R$, Walton $M A$, et al. Alcohol use severity and age moderate the effects of brief interventions in an emergency department randomized controlled trial. Drug Alcohol Depend 2019;194:386-94.

3. White $A M$, Slater $M E, N g$ G, Hingson $R$, Breslow R. Trends in alcohol-related emergency department visits in the United States: results from the nationwide emergency department sample, 2006 to 2014. Alcohol Clin Exp Res 2018;42:352-9.

4. Lee SM, Yoon YD, Baek JH, Hyun KR, Kang HR. Assessing the impact of socioeconomic impacts of major health risk factors and regulatory policies [Internet]. Seoul: National Health Insurance Service; 2015 [cited 2019 Apr 5]. Available from: https://www.nhis.or.kr/nhis/together/wbhaec07800m01. do? mode $=$ view \&tarticleNo $=116002$.

5. Statistics Korea. 2018 Report on the social survey [Internet]. Daejeon: Korean Statistical Information Service; 2018 [cited 
2019 Apr 5]. Available from: http://kostat.go.kr/portal/korea/ kor_nw/1/6/3/index.board?bmode $=$ read\&tbSeq $=\varepsilon t a S e q=371$ $501 \&$ tpageNo $=1 \&$ trow Num $=10 \mathrm{Enav}$ Count $=10 \mathrm{\& tcurrPg}=\& \mathrm{ts}$ earchlnfo $=$ etsTarget $=$ titlectsTxt $=$.

6. World Health Organization. Global status report on alcohol and health 2018 [Internet]. Geneva: World Health Organization; 2018 [cited 2019 Apr 5]. Available from: https://apps. who.int/iris/bitstream/handle/10665/274603/9789241565639eng.pdf?ua $=1$.

7. Ministry of Health and Welfare. The epidemiology survey of mental disorders in Korea. Seoul: Ministry of Health and Welfare; 2011.

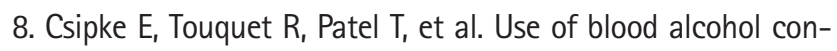
centration in resuscitation room patients. Emerg Med J 2007; 24:535-8.

9. Maier RV. Controlling alcohol problems among hospitalized trauma patients. J Trauma 2005;59:S1-2.

10. Fiellin DA, Reid MC, O'Connor PG. Screening for alcohol problems in primary care: a systematic review. Arch Intern Med 2000;160:1977-89.

11. Hingson R, Day N. Comment on "Alcoholism: a controlled trial of 'treatment' and 'advice'". J Stud Alcohol 1977;38:2206-11.

12. Bien TH, Miller WR, Tonigan JS. Brief interventions for alcohol problems: a review. Addiction 1993;88:315-35.

13. Schmidt CS, Schulte B, Seo HN, et al. Meta-analysis on the effectiveness of alcohol screening with brief interventions for patients in emergency care settings. Addiction 2016;111:78394.

14. Noh H, Jung KY, Park HS, Cheon YJ. Characteristics of alcoholrelated injuries in adolescents visiting the emergency department. J Korean Med Sci 2011;26:431-7.

15. Babor TF, Higgins-Biddle JC, Saunders JB, Monteiro MG. The alcohol use disorders identification test: guidelines for use in primary health care. Geneva: World Health Organization; 1992.

16. Rehm J, Gmel GE Sr, Gmel G, et al. The relationship between different dimensions of alcohol use and the burden of diseasean update. Addiction 2017;112:968-1001.
17. Academic ED SBIRT Research Collaborative. The impact of screening, brief intervention, and referral for treatment on emergency department patients' alcohol use. Ann Emerg Med 2007;50:699-710.

18. D'Onofrio G, Pantalon MV, Degutis LC, et al. Brief intervention for hazardous and harmful drinkers in the emergency department. Ann Emerg Med 2008;51:742-50.

19. van Loon $M$, van Gaalen ACP, van der Linden MC, HagesteinDe Bruijn C. Evaluation of screening and brief intervention for hazardous alcohol use integrated into clinical practice in an inner-city Emergency Department. Eur J Emerg Med 2017;24: 224-9.

20. Moyer A, Finney JW. Brief interventions for alcohol misuse. CMAJ 2015;187:502-6.

21. National Institute on Alcohol Abuse and Alcoholism. Helping patients who drink too much: a clinician's guide [Internet]. Bethesda, MD: National Institute on Alcohol Abuse and Alcoholism; 2005 [cited 2019 Apr 29]. Available from: https://pubs. niaaa.nih.gov/publications/practitioner/cliniciansguide2005/ guide.pdf.

22. Barata IA, Shandro JR, Montgomery M, et al. Effectiveness of SBIRT for alcohol use disorders in the emergency department: a systematic review. West J Emerg Med 2017;18:1143-52.

23. Daeppen JB, Gaume J, Bady P, et al. Brief alcohol intervention and alcohol assessment do not influence alcohol use in injured patients treated in the emergency department: a randomized controlled clinical trial. Addiction 2007;102:122433.

24. Neighbors CJ, Barnett NP, Rohsenow DJ, Colby SM, Monti PM. Cost-effectiveness of a motivational intervention for alcoholinvolved youth in a hospital emergency department. J Stud Alcohol Drugs 2010;71:384-94.

25. Barbosa C, Cowell A, Bray J, Aldridge A. The cost-effectiveness of alcohol screening, brief intervention, and referral to treatment (SBIRT) in emergency and outpatient medical settings. J Subst Abuse Treat 2015;53:1-8. 
Appendix 1. Alcohol use disorders identification test

\begin{tabular}{|c|c|c|c|c|c|}
\hline Questions & 0 & 1 & 2 & 3 & 4 \\
\hline 1. How often do you have a drink containing alcohol? & Never & Monthly or less & $\begin{array}{l}\text { 2-4 times a } \\
\text { month }\end{array}$ & $\begin{array}{l}\text { 2-3 times } \\
\text { a week }\end{array}$ & 4 or more times a week \\
\hline $\begin{array}{l}\text { 2. How many drinks containing alcohol do you have on a typical day when } \\
\text { you are drinking? }\end{array}$ & 1 or 2 & 3 or 4 & 5 or 6 & 7 to 9 & 10 or more \\
\hline $\begin{array}{l}\text { 3. How often do you have six or more drinks on one monthly almost occa- } \\
\text { sion? }\end{array}$ & Never & Less than Monthly & Monthly & Weekly & Daily or almost daily \\
\hline $\begin{array}{l}\text { 4. How often during the last year have you found that were not able to } \\
\text { stop drinking once you had started? }\end{array}$ & Never & Less than Monthly & Monthly & Weekly & Daily or almost daily \\
\hline $\begin{array}{l}\text { 5. How often during the last year have you failed to do what was normally } \\
\text { expected of you because of drinking? }\end{array}$ & Never & Less than Monthly & Monthly & Weekly & Daily or almost daily \\
\hline $\begin{array}{l}\text { 6. How often during the last year have you needed a first drink in the } \\
\text { morning to get yourself going after a heavy drinking session? }\end{array}$ & Never & Less than Monthly & Monthly & Weekly & Daily or almost daily \\
\hline $\begin{array}{l}\text { 7. How often during the last year have you had a feeling of guilt or re- } \\
\text { morse after drinking? }\end{array}$ & Never & Less than Monthly & Monthly & Weekly & Daily or almost daily \\
\hline $\begin{array}{l}\text { 8. How often during the last year have you been unable to remember what } \\
\text { happened the night before because of your drinking? }\end{array}$ & Never & Less than Monthly & Monthly & Weekly & Daily or almost daily \\
\hline 9. Have you or someone else been injured because of your drinking? & No & & $\begin{array}{l}\text { Yes, but not in } \\
\text { the last year }\end{array}$ & & Yes, during the last year \\
\hline \multirow[t]{2}{*}{$\begin{array}{l}\text { 10. Has a relative, friend, doctor, or other health care worker been concerned } \\
\text { about your drinking or suggested you cut down? }\end{array}$} & No & & $\begin{array}{l}\text { Yes, but not in } \\
\text { the last year }\end{array}$ & & Yes, during the last year \\
\hline & & & & & Total \\
\hline
\end{tabular}


Appendix 2. Brief intervention protocol

\section{Provide feedback}

"How are you doing? I would like to inform you of the results of the alcohol screening test. The questionnaire that you have just answered is the widely used Alcohol Use Disorder Identification Test developed by the WHO, which is very useful for identifying patients with alcohol use disorder. Based on your test results, you are in the risk drinker group." After examining the response of the patient, please make one of the following statements: "Is this something you did not expect?" "Were you surprised?" or "Is this what you were concerned about?"

"People who drink the same amount of alcohol as you only account for $15 \%$ of the total human population. Can you tell me any diseases that are associated with drinking disorders?" (Please encourage the patients to express themselves.) "Alcohol use disorder is known to increase the risk of diseases including hypertension, gastrointestinal bleeding, sleep disorders, depression, hemorrhagic stroke, and cirrhosis as well as the risk for further health deterioration. Considering that excessive alcohol consumption can cause various kinds of problems, I am concerned about your current drinking patterns. What do you think about it?" (Please wait for the patient's response.)

"Then, what do you say about the test results showing that you are in the risk drinker group?"

\section{Enhance motivation}

$\square$ What might be the advantage of drinking alcohol?

$\checkmark$ Then, what might be the disadvantage of drinking alcohol?

(Please keep asking questions until the patient runs out of opinions and listen to what they say!)

$\rightarrow$ (Summarize the patient's comments for the patient as follows:)

You think that drinking alcohol can be bad for you because

Also, you think that drinking alcohol can be bad for you because

Please make one of the following statements: "Then, what should you do from now on?" "What do you have to do from now on?" or "What do you think you have to do from now on?"

Let's say there is a scale of 0 to 10 points.

Please rate how ready you are to change your drinking habit?

$\square$ If the patient says "6 points or higher" (for example, "6 points"):

"Why do you choose ' 6 points' instead of 3 or 4 points?"

$\square$ Even if the patient says " 5 points or lower," respond positively (for example, "3 points"):

"This means that you think you need to change it, because you choose ' 3 points' instead of ' 0 ."' 


\section{Advice}

"You need to cut down on alcohol consumption. The commonly recommended alcohol consumption amount is three or fewer glasses for men and two or one glass for women in a session. Why don't you reduce alcohol consumption?" (Please encourage the patient to speak in detail, for instance,

"__ cups for week or at one session.)

"First, how much can you reduce your alcohol consumption for this period?" (Please encourage the patient to speak in detail, for instance, two weeks or a month.)

"Here is a record of what you have told me today. This is the alcohol consumption reduction pledge, which emphasizes the drinking abstinence goal. What is important here is that you agree to the pledge by your own choice. I hope that you will do your best to achieve the goal and recommend that you write a diary to keep track of your alcohol consumption during the period that you decide not to drink."

If the patient's AUDIT score is 16 points or higher, you will say:

"Why don't you continue to participate in an alcohol abstinence program to reduce your alcohol consumption?" (Please help the patient sign the letter of consent.)

"As part of the interim checkup to assess how well a patient is following the pledge, we will call you (in one week, one month, and three months) to ask whether you maintained alcohol abstinence. Thank you for your time. I hope that you will achieve your alcohol reduction and abstinence goal." 